\title{
Tratamento das rupturas irreparáveis do manguito rotador: Reconstrução capsular superior com aloenxerto de fáscia lata
}

\section{Treatment of Irreparable Rotator Cuff Tears: \\ Superior Capsular Reconstruction with Fascia Lata Allograft}

\author{
Rodrigo Alves Beraldo ${ }^{10}$ Mauro Emilio Conforto Gracitelli ${ }^{10} \quad$ Eduardo Angeli Malavolta10

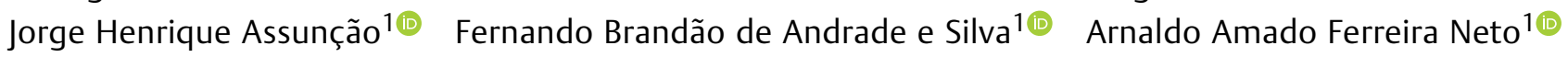 \\ 1 Ortopedista e Traumatologista, Especialista em Ombro e Cotovelo, \\ Instituto de Ortopedia e Traumatologia, Hospital das Clínicas, Faculdade \\ de Medicina, Universidade de São Paulo, São Paulo, SP, Brasil \\ Endereço para correspondência Rodrigo Alves Beraldo, MD, MSc, \\ Universidade de São Paulo, Rua Dr. Ovídio Pires de Campos, 333, $3^{\circ}$ \\ andar, Cerqueira Cesar, São Paulo, SP, 05403-010, Brasil \\ (e-mail: beraldo.ortop@gmail.com).
}

Rev Bras Ortop 2022;57(5):876-883.

\author{
Resumo

\section{Palavras-chave} \\ - lesões do manguito \\ rotador/cirurgia \\ - articulação do ombro \\ - cápsula articular \\ - fáscia \\ lata/transplante \\ - resultado do \\ tratamento
}

Objetivo O objetivo do presente estudo foi avaliar a eficácia e a segurança da reconstrução capsular superior (RCS) com a utilização do aloenxerto de fáscia lata. Métodos Uma série de casos prospectivos de 15 pacientes com ruptura irreparável do supraespinhal foi submetida a RCS com aloenxerto de fáscia lata, sendo adotada como desfecho primário a escala American Shoulder and Elbow Surgeons (ASES, na sigla em inglês) aos 12 meses do pós-operatório. Como desfechos secundários, foram adotadas as escalas da University of California Los Angeles (UCLA, na sigla em inglês), ConstantMurley, e Single Assessment Numeric Evaluation (SANE, na sigla em inglês), além da amplitude de movimento. Os parâmetros radiológicos também foram avaliados por radiografias simples e ressonância magnética (RM).

Resultados Quinze pacientes completaram 12 meses de acompanhamento pós-operatório. O escore ASES aumentou de 34,0 para 73,0 $(p=0,005)$. As escalas UCLA, ConstantMurley e SANE também apresentaram diferenças estatisticamente significativas $(p=0,001 ; p=0,005$; e $p=0,046)$. Na avaliação da amplitude de movimento, houve melhora na elevação e rotação externa ( 95 a $140^{\circ}, p=0,003 ; 30$ a $60^{\circ}, p=0,007$ ). Seis pacientes (40\%) tiveram cicatrização completa do enxerto. Os desfechos clínicos foram significativamente maiores nos pacientes que apresentaram cicatrização do enxerto.

\footnotetext{
Trabalho desenvolvido no Grupo de Ombro e Cotovelo do Instituto de Ortopedia e Traumatologia, Hospital das Clínicas, Faculdade de Medicina, Universidade de São Paulo, São Paulo, SP, Brasil.
}

recebido

21 de Outubro de 2020

aceito

11 de Fevereiro de 2021

Publicado on-line

Novembro 11, 2021
DOI https://doi.org/

$10.1055 / \mathrm{s}-0041-1732389$

ISSN 0102-3616.

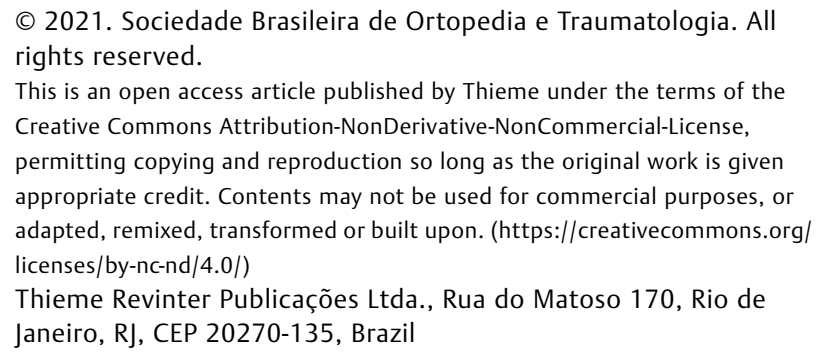

(c) 2021. Sociedade Brasileira de Ortopedia e Traumatologia. All rights reserved.

This is an open access article published by Thieme under the terms of the Creative Commons Attribution-NonDerivative-NonCommercial-License, permitting copying and reproduction so long as the original work is given appropriate credit. Contents may not be used for commercial purposes, or adapted, remixed, transformed or built upon. (https://creativecommons.org/ licenses/by-nc-nd/4.0/) Thieme Revinter Publicações Ltda., Rua do Matoso 170, Rio de Janeiro, RJ, CEP 20270-135, Brazil 


\author{
Abstract \\ Keywords \\ - rotator cuff \\ injuries/surgery \\ - shoulder joint \\ - joint capsule \\ - fascia \\ lata/transplantation \\ - treatment outcome
}

Conclusões A RCS com aloenxerto de fáscia lata é um procedimento seguro e eficaz com um curto acompanhamento de tempo. Nível de evidência IV; Estudo Terapêutico; Série de casos.

Objective The objective of the present study was to evaluate the efficacy and safety of superior capsular reconstruction (SCR) using fascia lata allograft.

Methods A prospective case series of 15 patients with irreparable supraspinatus tear who underwent SCR using fascia lata allograft. The American Shoulder and Elbow Surgeons (ASES) scale at 12 months after surgery was the primary outcome. The University of California Los Angeles (UCLA), Constant-Murley, and Single Assessment Numeric Evaluation (SANE) scales, in addition to the range of motion, were secondary outcomes. Radiological parameters were also evaluated by simple radiographs and magnetic resonance imaging (MRI).

Results Fifteen patients completed 12 months of postoperative follow-up. The ASES score increased from 34.0 to $73.0(p=0.005)$. The UCLA, Constant-Murley, and SANE scales also showed statistically significant differences $(p=0.001 ; p=0.005$; and $p=0.046)$. In the evaluation of range of motion, there was improvement in elevation and in external rotation ( 95 to $140^{\circ}, p=0.003 ; 30$ to $60^{\circ}, p=0.007$ ). Six patients ( $40 \%$ ) had complete graft healing. The clinical outcomes were significantly higher in the patients who presented graft healing.

Conclusions Superior capsular reconstruction using a fascia lata allograft is a safe and effective procedure in short follow-up. Level of Evidence IV; Therapeutic Study; Case Series.

\section{Introdução}

A ruptura do manguito rotador é uma condição comum que afeta $\sim 20 \%$ da população. ${ }^{1} 0$ tratamento cirúrgico para esta lesão geralmente apresenta melhora clínica significativa. ${ }^{2}$ No entanto, as falhas de cicatrização são frequentes, com novas rupturas em $27 \%$ dos casos. ${ }^{3}$ O tratamento da ruptura maciça do manguito rotador é particularmente um desafio, pois o reparo e sua consequente cicatrização são dificultados pela retração do tendão, pela atrofia muscular e pela degeneração gordurosa. ${ }^{3}$ Ainda não há consenso para o tratamento das rupturas irreparáveis do supraespinhal, principalmente em pacientes jovens.

Mihata et al. ${ }^{4,5}$ descreveram um procedimento de reconstrução capsular superior (RCS) em pacientes com rupturas maciças do supraespinhal, visando restaurar a estabilidade superior da articulação glenoumeral, restabelecendo assim a função remanescente do manguito rotador. Originalmente, a técnica usava enxerto de fáscia lata autóloga. ${ }^{4}$ Para reduzir a morbidade do paciente e o tempo cirúrgico, o enxerto de derme humana acelularizada ganhou popularidade com as cirurgias de RCS. ${ }^{6}$ Até o momento, nenhum estudo clínico usou o aloenxerto de fáscia lata para RCS. 0 uso desse enxerto teria algumas vantagens; entre elas, a diminuição da morbidade do paciente, o menor tempo cirúrgico e a adequada resistência do enxerto. ${ }^{7}$

O objetivo do presente estudo é avaliar os resultados clínicos e a cicatrização da RCS. Nossa hipótese é que a RCS com aloenxerto de fáscia lata apresenta bons resultados clínicos, principalmente quando há cicatrização do enxerto.

\section{Materiais e Métodos}

\section{Desenho do Estudo e a População Estudada}

Quinze pacientes foram submetidos a RCS em um estudo de coorte prospectivo. Todos os pacientes foram atendidos na mesma instituição e concordaram em assinar o termo de consentimento livre e esclarecido (CAAE62105616.4.0000.0068).

Foram incluídos pacientes com sintomas de dor, déficit funcional e diagnóstico de ruptura maciça do tendão supraespinhal, degeneração gordurosa classificada como grau 2, 3 ou 4, segundo Goutallier et al. ${ }^{8}$ Além disso, todos os pacientes realizavam rotação externa de forma ativa $>0^{\circ}$.

Não foram incluídos no presente estudo pacientes com ruptura subescapular de espessura total, com degeneração gordurosa tipo 3 ou 4, pacientes com artropatia do manguito rotador, considerada grau 5 pela classificação de Hamada et al., ${ }^{9}$ nem os pacientes com lesões anteriores do ombro, como as lesões do plexo braquial, fraturas proximais do úmero e com infecção tratada ou ativa. Foram excluídos os casos nos quais foi possível o reparo do tendão supraespinhal e os casos nos quais não foi possível o reparo do tendão subescapular.

\section{Intervenção}

As cirurgias foram realizadas pelo mesmo cirurgião de ombro e cotovelo com 11 anos de experiência [cego]. Foram utilizados aloenxertos de fáscia lata do banco de tecidos, conforme protocolo de coleta padrão da instituição.

0 procedimento seguiu as etapas descritas por Mihata et al., ${ }^{4,10}$ mas com a técnica de fixação de fileira simples, previamente descrita pelos autores [cego]. Todos os casos 


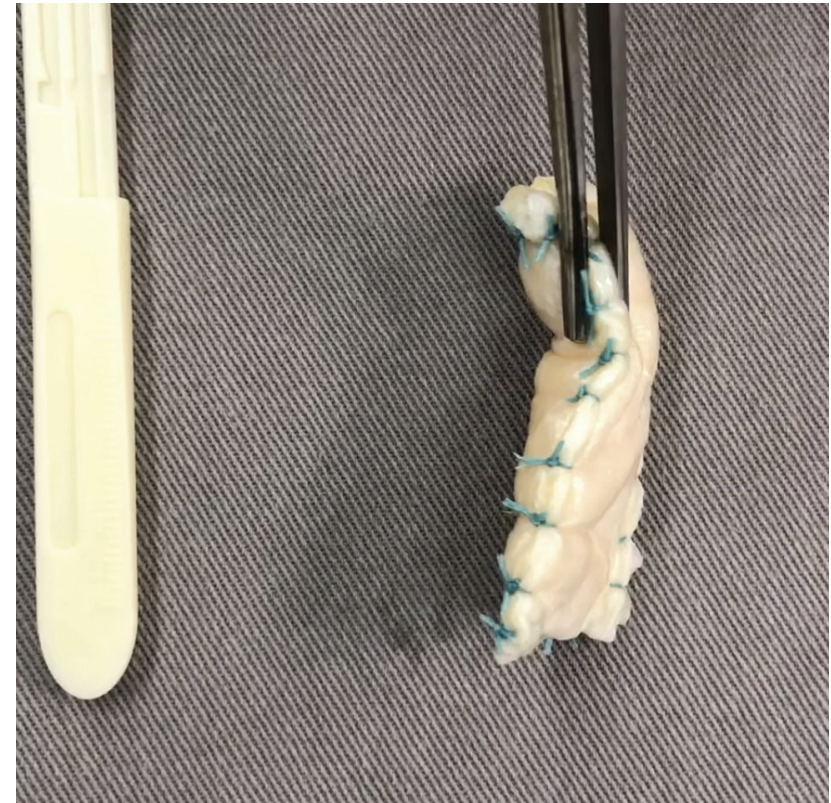

Fig. 1 Aloenxerto de fáscia lata com $8 \mathrm{~mm}$ de espessura.

foram operados pela técnica artroscópica, sendo o paciente posicionado em decúbito lateral com o braço abduzido a $30^{\circ}$. Em todos os casos, foram realizadas tenotomia do bíceps, bursectomia e acromioplastia. As rupturas subescapulares foram reparadas nos casos de ruptura parcial ou total. Os

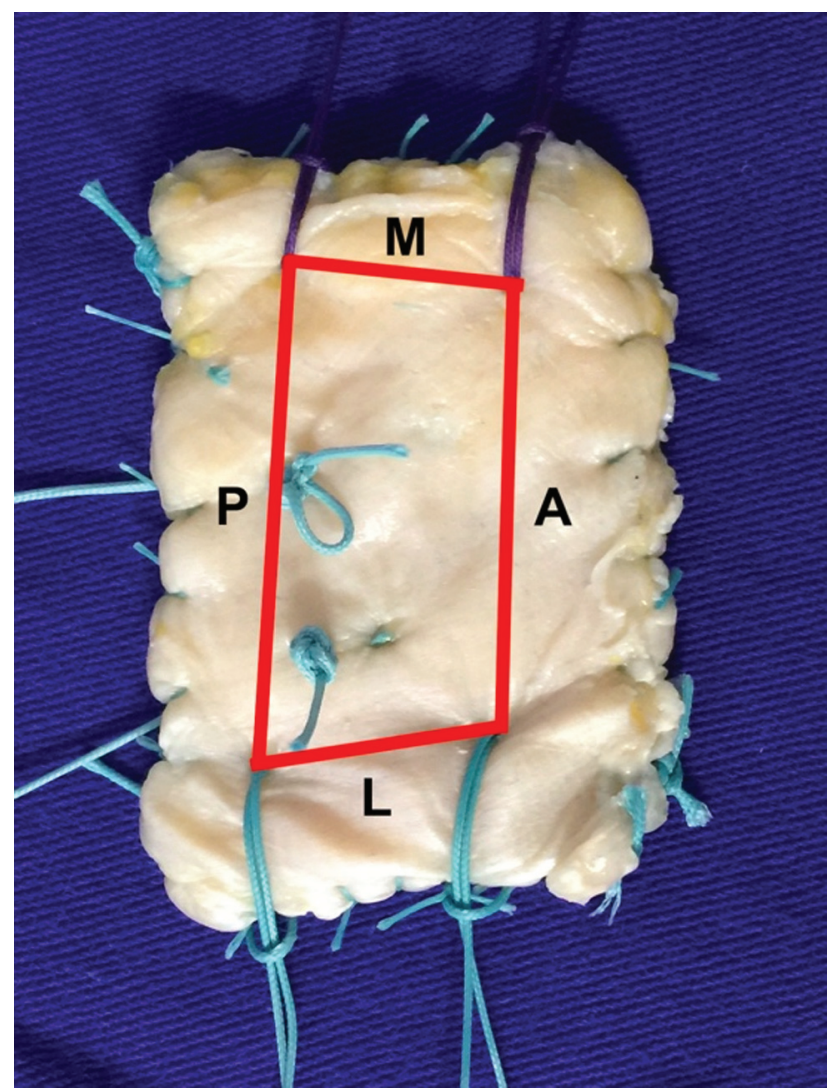

Fig. 2 Aloenxerto de fáscia lata com suturas provisórias, de acordo com as medidas tomadas artroscopicamente.

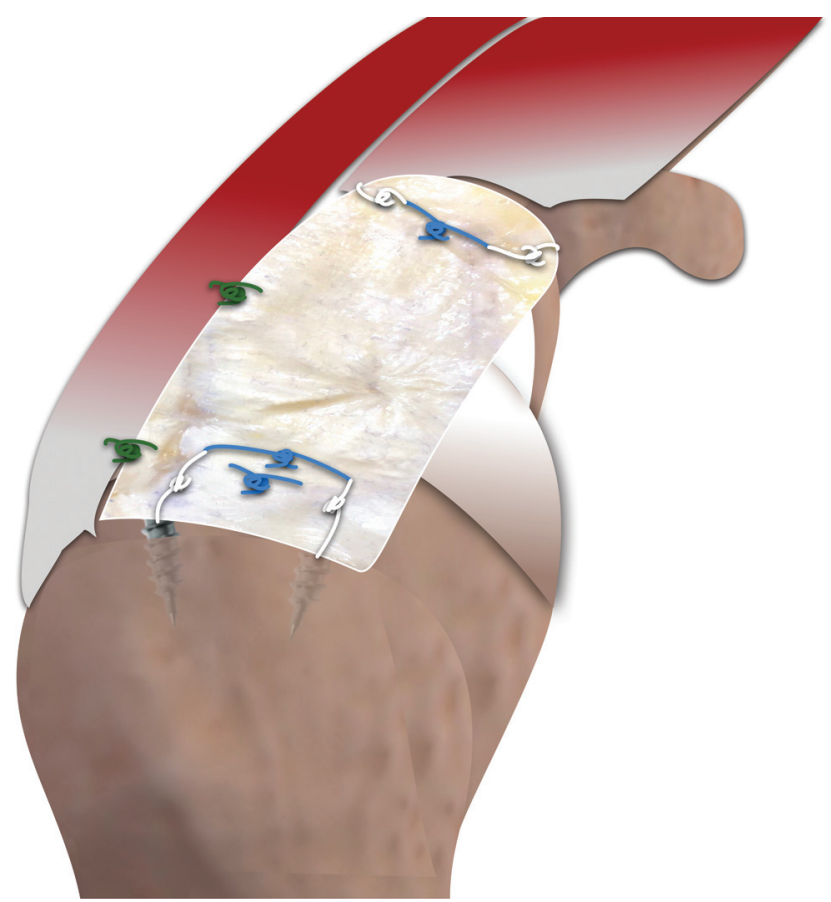

Fig. 3 Aspecto final da reconstrução capsular superior com aloenxerto de fáscia lata.

pacientes cujo tendão supraespinhal era passível de reparo foram excluídos do estudo.

A primeira etapa da RCS consistiu em preparar a região superior da glenóide e da tuberosidade maior, com a criação dos portais acessórios. Foram feitos dois portais para a inserção das âncoras no tubérculo maior, anterossuperior e posterossuperior. Também foi feito o portal de Neviaser, que permitiu a inserção da âncora posterior na glenóide, possibilitando a tração do enxerto por um sistema de dupla polia. A âncora anterior da glenóide foi introduzida através do portal anterior. Todas as âncoras utilizadas foram de $5 \mathrm{~mm}$, metálicas, e de dupla carga.

Em seguida, foi preparado um enxerto de tamanho adequado, tendo sido dobrado de 3 a 4 vezes, a fim de atingir a espessura de 6 a $8 \mathrm{~mm}$ (-Fig. 1). As distâncias entre as âncoras foram utilizadas para determinar a localização das suturas do enxerto (-Fig. 2).

A etapa subsequente consistiu na fixação do enxerto na glenóide, por meio de sutura com dupla polia de fora para dentro, na qual o enxerto foi tracionado pelos fios da âncora através do portal medial de Neviaser. Após a fixação medial e lateral do enxerto, foram realizadas suturas simples entre o enxerto e o tendão infraespinhal (-Fig. 3). ${ }^{11}$

\section{Acompanhamento Pós-operatório}

A reabilitação seguiu o mesmo protocolo do reparo do manguito rotador. Os pacientes foram orientados a usar uma tipoia com almofada para abdução por 6 semanas. Após quatro semanas, os pacientes foram submetidos à terapia de movimento passivo. Depois da 6 $6^{\underline{a}}$ semana, foi iniciada a terapia de movimento ativo. Doze semanas após a cirurgia, foram iniciados os exercícios de fortalecimento do 
manguito rotador e da musculatura estabilizadora da escápula.

\section{Desfechos}

A escala ASES aplicada aos 12 meses após a cirurgia foi adotada como desfecho primário. Os desfechos secundários adotados foram as escalas Constant-Murley, UCLA e SANE, sendo que a amplitude de movimento ativa foi avaliada com o paciente na posição ortostática. Foram avaliadas a elevação, a rotação externa e a interna. Todas as medidas foram realizadas com goniômetro, exceto a rotação interna, que foi avaliada de acordo com a posição da mão do paciente em relação aos processos espinhosos vertebrais, sendo posteriormente convertidos para números contínuos, por meio de uma escala de 1 a 19 (T1 a T12 = 1 a $12, \mathrm{~L} 1$ a $\mathrm{L} 5=13$ a 17 , sacro = 18, e trocanter maior $=19$ ). A distância entre o acrômio e a cabeça do úmero foi avaliada pelo método de Ellman, ${ }^{12}$ e a avaliação da cicatrização do enxerto foi feita por meio de RM, realizada 6 meses após o procedimento; assim como as complicações e as reintervenções cirúrgicas. 0 enxerto foi considerado "não cicatrizado" quando apresentava sinais de descontinuidade em dois ou mais cortes coronais oblíquos e contínuos em T1 e T2. As escalas e a amplitude de movimento foram avaliadas por um avaliador independente, e as RMs foram avaliadas por um radiologista musculoesquelético.

\section{Análise Estatística}

Submetemos as variáveis contínuas à avaliação da normalidade pelo teste de Kolmogorov-Smirnov e da homogeneidade pelo teste de Levene. Os dados contínuos foram expressos em média, mediana, desvio padrão(DP) e intervalo interquartil. As variáveis categóricas foram expressas em valor absoluto e porcentagem. A comparação entre as diferentes variáveis antes e depois do tratamento foi realizada com o teste qui-quadrado ou exato de Fisher para as variáveis categóricas. O teste de Wilcoxon foi utilizado para variáveis contínuas. Utilizou-se o programa IBM SPSS Statistics for Windows versão 21.0 (IBM Corp., Armonk, NY, EUA) para análise dos dados, com nível de significância de $5 \%$.

\section{Resultados}

Vinte pacientes foram inicialmente incluídos para RCS com o uso do aloenxerto de fáscia lata entre março de 2017 e fevereiro de 2018. Durante a cirurgia, 5 pacientes foram excluídos do estudo após reparo completo do manguito rotador. Nenhum paciente foi perdido para o acompanhamento. Assim, 15 pacientes receberam o tratamento cirúrgico proposto. As características gerais da amostra são apresentadas na - Tabela 1.

A mediana da escala ASES passou de um valor préoperatório de 34,0 para 73,0 em 12 meses $(p=0,005)$. A escala UCLA passou de 11,0 para 27,0, a Constant-Murley de 41,0 para 73,0 , e a SANE de 50,0 para $70,0(p=0,001$, $p=0,005$ e $p=0,046$, respectivamente). Os valores são mostrados na - Tabela 2.

Os pacientes apresentaram diferença estatisticamente significativa para a amplitude de movimento ativa, tanto
Tabela 1 Distribuição das frequências absolutas e relativas das variáveis intrínsecas ao paciente

\begin{tabular}{|c|c|}
\hline & Casos \\
\hline \multicolumn{2}{|l|}{ Gênero, n (\%) } \\
\hline Masculino & $8(53,3)$ \\
\hline Feminino & $7(46,7)$ \\
\hline \multicolumn{2}{|l|}{ Idade (anos) } \\
\hline Média (DP) & $60.6(8,56)$ \\
\hline \multicolumn{2}{|c|}{ Tabagismo, n (\%) } \\
\hline Sim & $1(6,7)$ \\
\hline Ex-tabagista & $4(26,7)$ \\
\hline Não & $11(66,6)$ \\
\hline \multicolumn{2}{|l|}{ Diabetes, n (\%) } \\
\hline Sim & $4(26,7)$ \\
\hline Não & $11(73,3)$ \\
\hline \multicolumn{2}{|c|}{ Lado acometido, n (\%) } \\
\hline Direito & $13(86,7)$ \\
\hline Esquerdo & $2(13,3)$ \\
\hline \multicolumn{2}{|c|}{ Lado dominante acometido, n (\%) } \\
\hline Sim & $15(100)$ \\
\hline Não & $0(0)$ \\
\hline \multicolumn{2}{|c|}{ Cirurgia prévia, n (\%) } \\
\hline Sim & $3(20)$ \\
\hline Não & $12(80)$ \\
\hline
\end{tabular}

Abreviações: DP, desvio padrão; n, número de pacientes.

na elevação $(p=0,003)$ quanto na rotação externa $(p=0,007)$. Os valores são mostrados na - Tabela 3.

Houve aumento da distância acromioumeral média de 3,62 $\mathrm{mm}(\mathrm{DP}=1,4)$ para 7,04 $\mathrm{mm}(\mathrm{DP}=1,6)$.

A cicatrização do enxerto ocorreu em 6 pacientes (40\%), evidenciada por RM pós-operatória aos 6 meses. Entre os pacientes sem cicatrização, houve predomínio de Hamada 3 e 4 (66,7\%), enquanto entre aqueles com cicatrização, houve predomínio de Hamada 1 e 2 (83,3\%). A - Tabela 4 mostra a distribuição dos pacientes, comparando a classificação de Hamada pré-operatória e a cicatrização do enxerto.

$\mathrm{Na}$ análise do subgrupo comparando os casos com e sem cicatrização do enxerto, ficou demonstrado que os casos com cicatrização apresentaram melhores resultados funcionais de acordo com as escalas ASES, UCLA e Constant-Murley, além de uma maior elevação ativa e rotação interna. Os valores são apresentados na - Tabela 5 e na -Tabela 6.

Não foram relatadas infecções superficiais ou profundas, lesões neurológicas, hematomas ou rigidez articular.

\section{Discussão}

Em relação aos desfechos clínicos, houve melhora estatisticamente significativa na escala ASES, com pontuação pré-operatória mediana de 34,0 e escore em 12 meses de 
Tabela 2 Resultados da avaliação clínica de acordo com as escalas ASES, UCLA, Constant-Murley e SANE

\begin{tabular}{|c|c|c|}
\hline & Mediana & valor-p \\
\hline \multicolumn{3}{|l|}{ ASES } \\
\hline Inicial & 34 & \\
\hline 6 meses & 72 & 0,008 \\
\hline 12 meses & 73 & 0,005 \\
\hline \multicolumn{3}{|l|}{ UCLA } \\
\hline Inicial & 11 & \\
\hline 6 meses & 24 & 0,005 \\
\hline 12 meses & 27 & 0,001 \\
\hline \multicolumn{3}{|c|}{ Constant-Murley } \\
\hline Inicial & 41 & \\
\hline 6 meses & 66 & 0,017 \\
\hline 12 meses & 73 & 0,005 \\
\hline \multicolumn{3}{|l|}{ SANE } \\
\hline Inicial & 50 & \\
\hline 6 meses & 60 & 0,231 \\
\hline 12 meses & 70 & 0,046 \\
\hline
\end{tabular}

Abreviações: ASES, American Shoulder and Elbow Surgeons; SANE, Single Assessment Numeric Evaluation; UCLA, University of California, Los Angeles.

Tabela 3 Avaliação da amplitude de movimento ativa

\begin{tabular}{|c|c|c|}
\hline & Mediana & valor-p \\
\hline \multicolumn{3}{|l|}{ Elevação } \\
\hline Inicial & 95,0 & \\
\hline 6 meses & 140,0 & 0,044 \\
\hline 12 meses & 140,0 & 0,003 \\
\hline \multicolumn{3}{|c|}{ Rotação externa } \\
\hline Inicial & 30,0 & \\
\hline 6 meses & 40,0 & 0,076 \\
\hline 12 meses & 60,0 & 0,007 \\
\hline \multicolumn{3}{|c|}{ Rotação interna } \\
\hline Inicial & 10,0 & \\
\hline 6 meses & 10,0 & 0,398 \\
\hline 12 meses & 10,0 & 0,624 \\
\hline
\end{tabular}

valor-p: nível de significância; *A rotação interna foi convertida em números contínuos. T1 a T12 equivale aos valores de 1 a 12, L1 a L5 aos valores de 13 a 17, o sacro equivale a 18 e o trocanter maior a 19 .

$73,0$ ( $p=0,003)$. Embora todos os estudos mostrem melhorias estatisticamente significativas, existem algumas diferenças nos valores da escala ASES em comparação com os outros. Mihata et al., ${ }^{4}$ utilizando autoenxerto, obtiveram resultados excelentes com o escore médio da escala ASES, passando de 23,5 para 92,9 em seu primeiro estudo clínico publicado. Além disso, os mesmos autores relataram resul-
Tabela 4 Relação entre a cicatrização do enxerto e a classificação de Hamada ${ }^{16}$

\begin{tabular}{|l|l|l|l|l|}
\hline \multirow{2}{*}{} & \multicolumn{2}{|l|}{ Não cicatrizado } & \multicolumn{2}{l|}{ Cicatrizado } \\
\cline { 2 - 5 } & $\boldsymbol{n}$ & $\%$ & $\boldsymbol{n}$ & $\%$ \\
\hline HAMADA & & & & \\
\hline $1+2$ & 3 & 37,5 & 5 & 62,5 \\
\hline 3 & 2 & 66,7 & 1 & 33,3 \\
\hline 4 & 4 & 100,0 & 0 & 0,0 \\
\hline
\end{tabular}

Abreviação: n, número de pacientes.

tados semelhantes em outros estudos publicados posteriormente. ${ }^{13,14}$ No entanto, Pennington et al. ${ }^{15}$ e Burkhart et al. ${ }^{16}$ publicaram resultados promissores com o uso da derme acelular humana, porém sem atingir uma pontuação média na escala ASES $>90$ pontos (82 e 89, respectivamente). Semelhante ao nosso estudo, tanto Denard et al. ${ }^{17}$ quanto Hirahara et al. ${ }^{18}$ obtiveram resultados estatisticamente significantes, com valores da escala ASES $<80$ pontos (77,5 e 70,7, respectivamente), utilizando o enxerto dérmico. A melhora em todos os escores clínicos corrobora os resultados apresentados em quatro revisões da literatura publicadas recentemente. $^{19-22}$

Na avaliação da amplitude de movimento ativa, houve melhora tanto na elevação, que passou de 95 para $140^{\circ}$ $(p=0,003)$, quanto na rotação externa, que passou de 30 para $60^{\circ}(p=0,007)$. Nossos resultados estão de acordo com 0 que já foi publicado em relação à amplitude de movimento após a RCS, tanto em estudos que utilizaram enxerto autó$\operatorname{logo}_{0}^{4,13,23}$ quanto em estudos que utilizaram enxerto da derme acelular humana. ${ }^{15-18}$

Em relação à distância acromioumeral, observamos uma média de 3,62 mm no pré-operatório e de 7,04 mm no pósoperatório. Esse achado é semelhante ao demonstrado em publicações anteriores, com ganho que varia de 2,6 a $5 \mathrm{~mm}$ após a cirurgia. ${ }^{4,15,18}$

Na avaliação da cicatrização do enxerto após 6 meses de acompanhamento, 6 pacientes (40\%) apresentaram cicatrização completa, sendo que 9 pacientes (60\%) apresentaram sinais de não cicatrização. Da mesma forma, em 2018, Denard et al. ${ }^{17}$ publicaram uma taxa de cicatrização de $45 \%$, enquanto AlRamadhan et al. ${ }^{24}$ e Lee et al. ${ }^{25}$ relataram taxas de 69,3\% e 63,8\%, respectivamente. No entanto, Mihata et al. ${ }^{4,13}$ observaram taxas mais elevadas após a RCS, com níveis de 83,3 e de 93\% em estudos publicados em 2013 e 2015. Após uma revisão sistemática que avaliou um total de 350 casos, Catapano et al. ${ }^{19}$ relataram uma taxa de cicatrização do enxerto de $87,5 \%$, achado semelhante aos $85,8 \%$ publicados por Sochacki et al., ${ }^{20}$ também em revisão sistemática.

Quando correlacionamos a taxa de cicatrização do enxerto com a classificação de Hamada pré-operatória, observamos que dos 9 pacientes que não apresentaram cicatrização, 6 $(66,7 \%)$ foram classificados como Hamada 3 ou 4 , enquanto 83,3\% dos pacientes que apresentaram cicatrização foram classificados como Hamada 1 ou 2. Embora nossa amostra 
Tabela 5 Análise do subgrupo para as escalas ASES, UCLA, Constant-Murley e SANE, de acordo com a cicatrização do enxerto

\begin{tabular}{|c|c|c|c|c|c|}
\hline \multirow[t]{2}{*}{ Cicatrização } & NÃO & & SIM & & \\
\hline & Média & Mediana & Média & Mediana & valor-p \\
\hline \multicolumn{6}{|l|}{ ASES } \\
\hline Inicial & 36,1 & 37,0 & 39,0 & 31,5 & 0,906 \\
\hline 6 meses & 48,1 & 43,0 & 86,5 & 90,0 & 0,003 \\
\hline 12 meses & 58,0 & 55,0 & 87,3 & 90,0 & 0,013 \\
\hline \multicolumn{6}{|l|}{ UCLA } \\
\hline Inicial & 10,5 & 10,0 & 13,8 & 14,5 & 0,238 \\
\hline 6 meses & 16,1 & 15,0 & 29,3 & 30,0 & 0,011 \\
\hline 12 meses & 19,7 & 25,0 & 30,6 & 30,0 & 0,013 \\
\hline \multicolumn{6}{|c|}{ Constant-Murley } \\
\hline Inicial & 38,0 & 38,0 & 54,3 & 60,5 & 0,195 \\
\hline 6 meses & 44,7 & 37,0 & 74,8 & 80,0 & 0,007 \\
\hline 12 meses & 53,3 & 65,0 & 75,1 & 78,5 & 0,011 \\
\hline \multicolumn{6}{|l|}{ SANE } \\
\hline Inicial & 57,7 & 50,0 & 38,3 & 35,0 & 0,210 \\
\hline 6 meses & 53,3 & 50,0 & 80,0 & 80,0 & 0,023 \\
\hline 12 meses & 66,6 & 70,0 & 81,6 & 85,0 & 0,188 \\
\hline
\end{tabular}

Abreviações: ASES, American Shoulder and Elbow Surgeons; SANE, Single Assessment Numeric Evaluation; UCLA, University of California, Los Angeles.

Tabela 6 Avaliação da amplitude de movimento ativa de acordo com a cicatrização do enxerto

\begin{tabular}{|c|c|c|c|}
\hline & Não cicatrizado & Cicatrizado & valor-p \\
\hline \multicolumn{4}{|c|}{ Elevação (mediana ${ }^{\circ}$ ) } \\
\hline Inicial & 90 & 148 & 0,288 \\
\hline 12 meses & 133 & 175 & 0,020 \\
\hline \multicolumn{4}{|c|}{ Rotação externa (medianaº } \\
\hline Inicial & 20 & 47,5 & 0,121 \\
\hline 12 meses & 60 & 67,5 & 0,159 \\
\hline \multicolumn{4}{|c|}{ Rotação interna (mediana ${ }^{\circ}$ ) } \\
\hline Inicial & 12 & 8,5 & 0,184 \\
\hline 12 meses & 13 & 7 & 0,001 \\
\hline
\end{tabular}

tenha sido pequena, o que diminui o poder dos resultados, nossos dados corroboram os de Denard et al., ${ }^{17}$ que publicaram uma taxa de cicatrização de $75 \%$ nos pacientes Hamada 1 e 2, e de apenas 44\% nos pacientes classificados como Hamada 3 ou 4. Esses dados podem nos ajudar a entender as elevadas taxas de cicatrização publicadas por Mihata et al. ${ }^{4}(83,3 \%)$, visto que apenas 2 pacientes em sua série de 24 casos foram classificados como Hamada 3 ou 4.

Ao avaliarmos os escores clínicos pelos subgrupos "cicatrizados" e "não cicatrizados", observamos que eles não apresentaram diferença estatística pré-operatória. No entanto, no acompanhamento de 12 meses, todas as pontuações foram estatisticamente mais elevadas no grupo de cicatrização, exceto para a escala SANE, achados semelhantes aos descritos por Denard et al., ${ }^{17}$ que publicaram pontuação média do ASES de 90,0 em pacientes com cicatrização do enxerto versus 66,5 em casos sem cicatrização ( $p=0,027)$. Também observamos diferença estatisticamente significativa na avaliação da elevação ativa dos pacientes, com mediana de $175^{\circ}$ no subgrupo cicatrizado versus $133^{\circ}$ no subgrupo não cicatrizado ( $p=0,020)$, achado semelhante ao publicado por Mihata et al. ${ }^{13}$ Esses dados sugerem que a classificação de Hamada pré-operatória, pode auxiliar no prognóstico e na indicação do melhor procedimento de RCS. Frank et al., ${ }^{26}$ em revisão de literatura, sugeriram que apenas os pacientes classificados como Hamada 1 e 2 são candidatos à RCS, e nosso estudo corrobora essa sugestão.

Como limitações, podemos destacar o fato do presente estudo não ser comparativo com uma amostra relativamente 
pequena e um curto período de acompanhamento. No entanto, o tamanho da amostra está dentro da faixa média dos outros estudos, $4,16,18$ e o tempo de acompanhamento foi semelhante ao publicado em estudos anteriores. ${ }^{15-18,24}$ Além disso, outra limitação foi a fixação lateral do enxerto, realizada com a técnica de fileira simples. No entanto, bons resultados foram alcançados com a técnica de fileira simples, principalmente em pacientes com classificação 1 ou 2 de Hamada. ${ }^{24,25}$

Apesar de apresentar resultados iniciais satisfatórios, a RCS é um procedimento relativamente recente. Mais estudos clínicos são necessários para confirmar sua eficácia em longo prazo e definir o tipo certo de paciente para esta cirurgia. Além disso, o enxerto ideal e seu método de fixação também precisam ser definidos.

\section{Conclusão}

A RCS com aloenxerto de fáscia lata é um procedimento seguro que proporciona melhora clínica aos pacientes. Melhores resultados são demonstrados nos casos em que ocorre a cicatrização completa do enxerto.

\section{Suporte Financeiro}

Não houve suporte financeiro de fontes públicas, comerciais, ou sem fins lucrativos.

\section{Contribuições dos autores}

Cada autor contribuiu individual e significativamente para o desenvolvimento deste artigo:

$\mathrm{RAB}$ : redigiu o artigo; realizou as cirurgias; coleta de dados; aprovou a versão final.

MECG: redação do artigo; realizou as cirurgias; acompanhamento dos pacientes; aprovou a versão final.

EAM: análise estatística; revisão bibliográfica; aprovou a versão final.

JHA: interpretou os resultados do estudo; revisão bibliográfica; exame clínico; aprovou a versão final.

FBAS: coleta de dados; levantamento dos prontuários médicos; acompanhamento dos pacientes; aprovou a versão final.

AAFN: participou do processo de revisão; exame clínico; aprovou a versão final.

\section{Conflito de Interesses}

Os autores declaram não haver conflito de interesses.

\section{Referências}

1 Yamamoto A, Takagishi K, Osawa T, et al. Prevalence and risk factors of a rotator cuff tear in the general population. J Shoulder Elbow Surg 2010;19(01):116-120

2 Burks RT, Crim J, Brown N, Fink B, Greis PE. A prospective randomized clinical trial comparing arthroscopic single- and double-row rotator cuff repair: magnetic resonance imaging and early clinical evaluation. Am J Sports Med 2009;37(04): 674-682

3 McElvany MD, McGoldrick E, Gee AO, Neradilek MB, Matsen FA 3rd. Rotator cuff repair: published evidence on factors associated with repair integrity and clinical outcome. Am J Sports Med 2015; 43(02):491-500
4 Mihata T, Lee TQ Watanabe C, et al. Clinical results of arthroscopic superior capsule reconstruction for irreparable rotator cuff tears. Arthroscopy 2013;29(03):459-470

5 Mihata T, McGarry MH, Pirolo JM, Kinoshita M, Lee TQ. Superior capsule reconstruction to restore superior stability in irreparable rotator cuff tears: a biomechanical cadaveric study. Am J Sports Med 2012;40(10):2248-2255

6 Tokish JM, Beicker C. Superior capsule reconstruction technique using an acellular dermal allograft. Arthrosc Tech 2015;4(06): e833-e839

7 Mihata T, Bui CNH, Akeda M, et al. A biomechanical cadaveric study comparing superior capsule reconstruction using fascia lata allograft with human dermal allograft for irreparable rotator cuff tear. J Shoulder Elbow Surg 2017;26(12):2158-2166

8 Goutallier D, Postel JM, Bernageau J, Lavau L, Voisin MC. Fatty muscle degeneration in cuff ruptures. Pre- and postoperative evaluation by CT scan. Clin Orthop Relat Res 1994;(304):78-83

9 Hamada K, Fukuda H, Mikasa M, Kobayashi Y. Roentgenographic findings in massive rotator cuff tears. A long-term observation. Clin Orthop Relat Res 1990;(254):92-96

10 Mihata T, McGarry MH, Kahn T, Goldberg I, Neo M, Lee TQ. Biomechanical Effects of Acromioplasty on Superior Capsule Reconstruction for Irreparable Supraspinatus Tendon Tears. Am J Sports Med 2016;44(01):191-197

11 Gracitelli MEC, Beraldo RA, Malavolta EA, Assunção JH, Oliveira DRO, Ferreira Neto AA. Superior capsular reconstruction with fascia lata allograft for irreparable supraspinatus tendon tears. Rev Bras Ortop (Sao Paulo) 2019;54(05):591-596

12 Ellman H, Hanker G, Bayer M. Repair of the rotator cuff. End-result study of factors influencing reconstruction. J Bone Joint Surg Am 1986;68(08):1136-1144

13 Mihata T, Lee TQ. Clinical Outcomes of Superior Capsule Reconstruction for Irreparable Rotator Cuff Tears without Osteoarthritis in the Glenohumeral Joint. Journal of Shoulder and Elbow Surgery 2015;24(04):e107-e109

14 Mihata T, Lee TQ Fukunishi K, et al. Return to sports and physical work after arthroscopic superior capsule reconstruction among patients with irreparable rotator cuff tears. Am J Sports Med 2018;46(05):1077-1083

15 Pennington WT, Bartz BA, Pauli JM, Walker CE, Schmidt W. Arthroscopic superior capsular reconstruction with acellular dermal allograft for the treatment of massive irreparable rotator cuff tears: short-term clinical outcomes and the radiographic parameter of superior capsular distance. Arthroscopy 2018;34 (06):1764-1773

16 Burkhart SS, Hartzler RU. Superior capsular reconstruction reverses profound pseudoparalysis in patients with irreparable rotator cuff tears and minimal or no glenohumeral arthritis. Arthroscopy 2019;35(01):22-28

17 Denard PJ, Brady PC, Adams CR, Tokish JM, Burkhart SS. Preliminary results of arthroscopic superior capsule reconstruction with dermal allograft. Arthroscopy 2018;34(01):93-99

18 Hirahara AM, Andersen WJ, Panero AJ. Superior capsular reconstruction: clinical outcomes after minimum 2-year follow-up. Am J Orthop (Belle Mead NJ) 2017;46(06):266-278

19 Catapano M, de Sa D, Ekhtiari S, Lin A, Bedi A, Lesniak BP. Arthroscopic superior capsular reconstruction for massive, irreparable rotator cuff tears: a systematic review of modern literature. Arthroscopy 2019;35(04):1243-1253

20 Sochacki KR, McCulloch PC, Lintner DM, Harris JD. Superior capsular reconstruction for massive rotator cuff tear leads to significant improvement in range of motion and clinical outcomes: a systematic review. Arthroscopy 2019;35(04):1269-1277

21 Ekhtiari S, Adili AF, Memon M, et al. Sources, quality, and reported outcomes of superior capsular reconstruction: a systematic review. Curr Rev Musculoskelet Med 2019;12(02):173-180 
22 Galvin JW, Kenney R, Curry EJ, et al. Superior capsular reconstruction for massive rotator cuff tears: a critical analysis review. JBJS Rev 2019;7(06):e1; 1-11

23 Mihata T, Lee TQ, Hasegawa A, et al. Arthroscopic superior capsule reconstruction can eliminate pseudoparalysis in patients with irreparable rotator cuff tears. Am J Sports Med 2018;46(11):2707-2716

24 Al Ramadhan H, Sungjoon L, In-Ho J. Early MRI findings of arthroscopic superior capsule reconstruction (ASCR): how to prevent early failure. Arthroscopy 2017;33(06 Suppl):e21
25 Lee SJ, Min YK. Can inadequate acromiohumeral distance improvement and poor posterior remnant tissue be the predictive factors of re-tear? Preliminary outcomes of arthroscopic superior capsular reconstruction. Knee Surg Sports Traumatol Arthrosc 2018;26(07):2205-2213

26 Frank RM, Cvetanovich G, Savin D, Romeo AA. Superior capsular reconstruction: indications, techniques, and clinical outcomes. JBJS Rev 2018;6(07):e10; 1-8 